\title{
Avaliação da estabilidade de estocagem de ligante asfáltico modificado com blenda de borra oleosa de petróleo e borracha de pneus
}

\author{
Asphalt binder storage stability modified with oily sludge oil blend and tire rubber
}

\author{
D. C. S. da Graça', E. H. Cavalcante ${ }^{2}$, G. Cardoso ${ }^{3}$ \\ ${ }^{1}$ PEQ - Programa de Pós-Graduação em Engenharia Química, ${ }^{2}$ Departamento de Engenharia Civil, ${ }^{3}$ Departamento \\ de Engenharia Química
}

Universidade Federal de Sergipe, CEP 49100-11, São Crsitóvão- Sergipe, Brasil1

e-mail: agracadcs@yahoo.com.bregiselia@ufs.br

(Recebido em 15 de junho de 2015; aceito em 29 de outubro de 2015)

\begin{abstract}
O desenvolvimento de novas tecnologias para minimizar os efeitos da ação de variações climáticas e da alta densidade de tráfego em pavimentos rodoviários vem sendo pesquisado nos últimos anos empregando-se macromoléculas como modificadores de ligante asfáltico, na busca por misturas asfálticas de alto desempenho. O objetivo deste estudo foi utilizar borra oleosa de fundo de tanque, oriunda de unidade de tratamento primário de petróleo, passivo de difícil disposição ambiental, como agente modificador do cimento asfáltico de petróleo, CAP 50/70, e avaliar a estabilidade de estocagem do ligante modificado, quanto à separação de fase, por meio de análise térmica e reológica. Devido à difícil dispersão da borra oleosa de petróleo no CAP 50/70, blenda de borracha de pneus inservíveis com borra oleosa de petróleo, na proporção $15 / 85 \%$ em massa, foi preparada e utilizada na proporção de $10 \%$ em massa, como agente modificador. Os resultados obtidos mostram que o referido ligante modificado atende às especificações brasileiras, no que se refere à temperatura de usinagem e compactação de misturas asfálticas do tipo CAUQ, empregadas em revestimentos de pavimento rodoviário. Contudo, observou-se a existência de separação de fase no ligante modificado.
\end{abstract}

Palavras chave: CAP, ligante asfáltico, borra oleosa

The development of new technologies to minimize the effects of climate change action and the high density of traffic on road pavement has been researched in recent years using macromolecules as asphalt binder modifier in the pursuit of getting high performance asphalt mixtures. The objective of this study was to use oily sludge tank background, coming from primary oil processing unit, difficult environmental disposal liabilities as modifier of asphalt cement petroleum, CAP 50/70, and evaluate the stability of storage of binder modified as to phase separation by means of heat and rheological analysis. Due to the difficult dispersion of oily sludge in CAP 50/70, blend rubber tires and oily sludge in the proportion $15 / 85 \%$ by mass was prepared and used in the proportion of $10 \%$ by mass, as agent modifier. The results show that the modified binder that meets the Brazilian specifications as to the temperature machining and compaction of hot mix asphalt - HMA type employed in road surface coverings. However phase separation was observed in the modified asphalt binder.

Keywords: CAP, asphalt binder, oily sludge

\section{INTRODUÇÃO}

O uso de ligante modificado, com polímero e aditivo químico, em misturas asfálticas para revestimento de pavimento rodoviário objetivando minimizar os efeitos da ação de variações climáticas, da alta densidade de tráfego e desta forma estender a vida útil do pavimento, reduzir custo de sua manutenção e restauração, tornou-se expressivo nas últimas décadas $[1,2,3]$.

$\mathrm{O}$ fato de estudos terem concluído que mistura de ligante modificado com polímero ser mais estável a temperatura elevada e mais flexível a baixa temperatura em relação à mistura asfáltica convencional, tornou esse tipo de material especialmente atrativo e empregado com sucesso em revestimento de pavimento de rodovias com elevado volume de tráfego, atendendo à segurança e conforto do usuário $[4,5]$. Contudo a relação entre a composição química do ligante asfáltico

$$
113310-1
$$


e as suas propriedades físicas e reológicas é complexa, o que faz com que a sua modificação pela adição de agentes poliméricos, borracha ou outros aditivos exija estudos que permitam prever a existência, ou não, de compatibilidade e estabilidade da mistura asfalto-modificante, fatores importantes para a sua estabilidade de estocagem [5, 6].

Da literatura, sabe-se que o ligante asfáltico é formado basicamente por quatro frações químicas: saturados; aromáticos; resinas e asfaltenos (SARA), sendo que as três primeiras constituem a porção maltênica e a última, a porção asfaltênica. A variação na proporção entre estas frações origina asfaltos com diferentes propriedades químicas, físicas e reológicas. As frações saturadas e aromáticas funcionam como plastificantes das resinas e dos asfaltenos, sólidos amorfos e de alto peso molecular [7].

A complexa composição dos ligantes asfálticos faz com que muitas substâncias usadas como modificadores possuam baixa solubilidade e/ou dispersão e propicie a separação de fases no ligante modificado, cujo mecanismo está relacionado às diferentes composições químicas e constituição físico-química do ligante asfáltico e do modificador. Contudo, estudos têm demonstrado que a borracha de pneus apresenta uma boa dispersão no ligante asfáltico e que é possível obter estabilidade de estocagem do ligante asfalto modificado com borracha de pneus e polímeros através da adição de estabilizantes químicos $[8,9]$.

O pneu é um produto essencial para a indústria automobilística. Contudo, seu material é não biodegradável e grande quantidade é descartada em depósitos que ocupam áreas extensas, causando prejuízos ao ambiente. Acredita-se que a solução mais promissora para os pneus inservíveis é reutilizá-lo como modificador de ligante asfáltico a serem empregados na obtenção de misturas asfálticas para revestimento de pavimento rodoviário [10]. Apesar do revestimento rodoviário com uso do ligante asfalto-borracha ter preço de mercado 30\% acima do convencional, para os seus fabricantes vale a pena o investimento, porque este chega a durar até três vezes mais, dependendo das condições climáticas e da carga de tráfego das rodovias [11]. O presente artigo apresenta estudos realizados sobre o uso de borracha de pneus inservíveis e borra oleosa de petróleo como agentes modificadores de ligante asfáltico.

A indústria petrolífera em todas as suas operações, desde a perfuração até a distribuição dos derivados, gera resíduos oleosos de diversos tipos. As refinarias respondem pela maior parte dos resíduos gerados, dos quais se destacam os resíduos oleosos acumulados no fundo dos tanques de óleo cru, lodos oleosos, lodos das torres de resfriamento, catalisadores gastos, resíduos das torres de trocadores de calor, finos de coque e águas residuais. Os resíduos oleosos de fundo de tanques, denominados borras oleosas de petróleo, são misturas multifásicas, compostas basicamente de emulsões água/óleo e sólidos (orgânicos e inorgânicos) que adsorvem o óleo, e por sedimentação formam resíduos ricos em frações pesadas de óleo cru e areia [12, 13].

Diante das considerações postas, o objetivo deste estudo foi avaliar o comportamento reológico e a estabilidade de estocagem do ligante asfáltico CAP 50/70 modificado com borra oleosa, advinda de tratamento primário de petróleo. Para tal, blenda de borracha de pneus inservíveis com borra oleosa, advinda de fundo de tanque do tratamento primário de petróleo, na proporção $15 / 85 \%$ em massa, respectivamente, foi obtida e adicionada ao ligante asfáltico em estudo, na concentração de $10 \%$ em massa.

\section{MATERIAL E MÉTODOS}

\section{Material}

Os materiais utilizados neste trabalho foram o ligante asfáltico de Petróleo CAP 50/70, cedido pela Empresa Municipal de Urbanismo da cidade de Aracaju, do Estado de Sergipe (EMURB - Aracaju/SE), a borra de petróleo como agente modificador de CAP, cedida pela Unidade de Operações de Exploração e Produção de Sergipe e Alagoas (UO - SEAL)/Petróleo Brasileiro S.A. - PETROBRAS, através da e a borracha de pneus inservíveis, adquirida de empresas de recauchutagem da cidade de Itabaiana/SE, na forma particulada. 


\section{Métodos}

Para a preparação da blenda, borracha de pneus inservíveis foi adicionada à borra oleosa de petróleo, nas respectivas proporções, 15/85 \% em massa (M1). A borra oleosa foi previamente aquecida a uma temperatura de $100 \pm 5^{\circ} \mathrm{C}$, durante 20 minutos, sob agitação mecânica de $40 \pm 2$ rpm, para o seu completo amolecimento. Em seguida foi adicionada borracha de pneu, na forma particulada, de diâmetro $\leq 32$ mesh, e realizada a mistura na temperatura de $160^{\circ} \mathrm{C}$, sob a mesma agitação, por mais 40 minutos, até completada dispersão. A blenda M1 obtida, após resfriada em bancada, a temperatura ambiente $\left(25^{\circ} \mathrm{C}\right)$, foi caracterizada por DSC, para avaliar a existência ou não de miscibilidade entre a borra oleosa de petróleo e a borracha de pneus inservíveis.

A obtenção do CAP 50/70 modificado ocorreu com a adição de $10 \%$ em massa da blenda $\mathrm{M} 1$, a $170 \pm 5^{\circ} \mathrm{C}$, sob agitação mecânica de $40 \pm 2 \mathrm{rpm}$, durante 90 minutos. Após a mistura, o CAP 50/70 modificado (CM1) foi vertido em artefato cilíndrico de aço inox, de $30 \mathrm{~mm}$ de diâmetro e $150 \mathrm{~mm}$ de altura e mantido em estufa de circulação de ar, na posição vertical, a 160 $\pm 5^{\circ} \mathrm{C}$, por 48 horas. Passado este tempo, o referido artefato de aço com a amostra foi retirado da estufa e submetido a resfriamento brusco, em banho de gelo e salmoura, e mantido por mais 2 horas em freezer, para preservar o histórico térmico da amostra, obtido durante o repouso de 48 horas na estufa de circulação de ar $[14,15]$. Em seguida, amostras foram retiradas da parte superior e inferior do artefato de aço e analisadas, comparativamente com o ligante asfáltico 50/70 não modificado, quanto ao comportamento da viscosidade com a taxa de cisalhamento, na temperatura de $60 \pm 0,1^{\circ} \mathrm{C}$, para averiguar a existência ou não de separação de fase.

A Figura 1 mostra o artefato utilizado para acondicionar as amostras durante o estudo de estabilidade de estocagem do CAP 50/70 modificado (CM1).

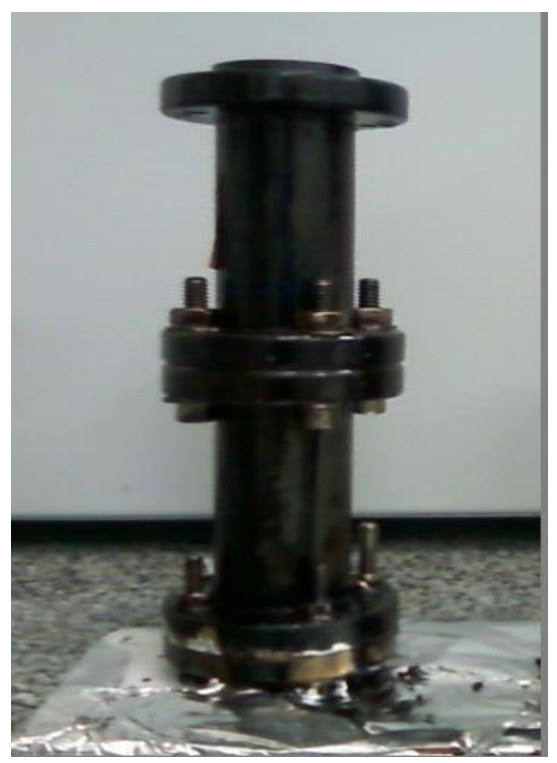

Figura 1 - Artefato de aço usado para estudar estabilidade de estocagem do CAP 50/70 modificado no LDCMat/UFS.

A energia de ativação de fluxo do CAP 50/70 puro e modificado foi determinada a partir da curva do logaritmo da viscosidade versus o inverso da temperatura em kelvin, obtida através do reômetro Brookfield, na faixa de temperatura de $175^{\circ}$ a $120 \pm 5^{\circ} \mathrm{C}$, com rotação de $35 \mathrm{rpm}$, taxa de resfriamento de $2,8^{\circ} \mathrm{C} / \mathrm{mim}$ e tempo de equilíbrio de 6 minutos, por meio da inclinação da curva da equação de Arrhenius modificada (Eq1).

$$
\eta=A e^{E_{f} / R T}
$$

Onde,

$\eta$ é a viscosidade dinâmica do líquido em cP

$\mathrm{E}_{\mathrm{f}}$ é a energia de ativação de fluxo em $\mathrm{J} / \mathrm{mol}$ 
R é constante dos gases $\left(8,314 \mathrm{~J}\right.$. $\left.\mathrm{mol}^{-1} \mathrm{~K}^{-1}\right)$

$\boldsymbol{A}$ é a constante que depende da natureza de cada fluido.

Análise reológica dinâmico-mecânica também foi realizada utilizando o reômetro de deformação controlada da TA Instruments Asphalt Rheometer CSA II com geometria de placas paralelas ( $25 \mathrm{~mm}$ de diâmetro) para o do CAP 50/70 puro e modificado CM1 na temperatura de $60^{\circ} \mathrm{C}$, a uma varredura de frequência de $0,1-100 \mathrm{~Hz}$, a uma amplitude fixa $1 \times 10^{-5} \mathrm{rad}$, gap de $1,0 \mathrm{~mm}$ e tempo de espera de 15 min para a coleta dos valores de G' e G'.

\section{RESULTADOS E DISCUSSÃO}

Comportamento térmico por DSC do CAP 50/70 puro e modificado e modificadores.

A Figura 2 mostra as curvas de resfriamento das amostras de CAP 50/70, borra oleosa de petróleo, borracha de pneu, blenda M1, e ligante modificado CM1. Observa-se que nenhum evento característico de cristalização aparece no CAP 50/70 e nem da borracha de pneu, na faixa de temperatura analisada, já que ambos são amorfos. Contudo, pico característico de cristalização é evidenciado na borra oleosa de petróleo, na blenda M1 e no ligante modificado CM1, de certo advindo da presença de fase cristalina presente na borra oleosa de petróleo [16, $17,18]$. Observa-se também que o referido pico de cristalização é deslocado para a direita na blenda M1 e ligeiramente para a esquerda, no ligante modificado CM1, indicando que a presença da borracha de pneu inservível facilita a cristalização da fase cristalina presente na borra oleosa de petróleo, enquanto que a presença do CAP 50/70 inibiu a cristalização da citada fase na amostra CM1, sinalizando em ambas as situações a existência de fase cristalina advinda da adição da borra oleosa de petróleo e que a sua presença também pode estar relacionada ao fenômeno de quimissorção entre o CAP 50/70 e componentes cristalizáveis presentes na borra oleosa $[19,20]$. A variação na intensidade dos picos pode ser atribuída ao menor volume da borra oleosa de petróleo presente na amostra e consequentemente menor quantidade de fase cristalina [21].

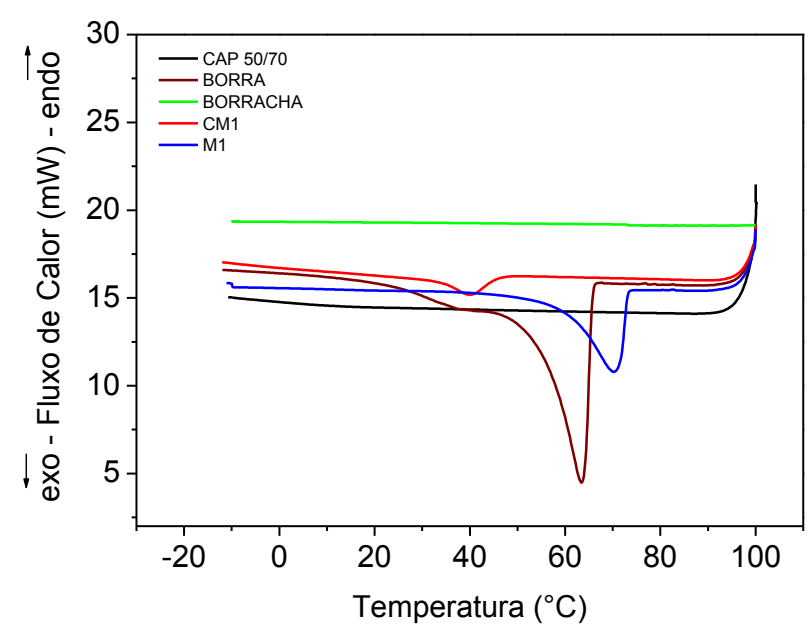

Figura 2 - Comportamento das amostras obtido por DSC em resfriamento do CAP 50/70 puro, borra oleosa de petróleo, borracha de pneu, blenda M1 e CAP 50/70 modificado CM1. 


\section{Determinação da energia de ativação}

A partir dos coeficientes angulares das curvas de viscosidade versus $1 / \mathrm{T}$ foram obtidos os dados da energia de ativação de fluxo $\left(\mathrm{E}_{\mathrm{f}}\right)$ apresentados na Tabela 1 , onde se observa uma menor energia de ativação de fluxo para o CAP 50/70 modificado, quando comparados ao do CAP 50/70 puro, o que confere um saldo positivo no balanço de energia quando da usinagem de misturas asfálticas para o revestimento de pavimento rodoviário utilizado.

Tabela 1 - Energia de ativação P 50/70 modificado, quando comparados ao do CAP 50/70 puro, o que confere um saldo de fluxo do CAP 50/70 puro e modificado

\begin{tabular}{c|c}
\hline MATERIAL & $\mathbf{E}_{\mathbf{f}}\left(\mathbf{K J} \mathbf{~ m o l}^{-1}\right)$ \\
\hline CAP 50/70 & 66,00 \\
CM1 & 62,65 \\
\hline
\end{tabular}

\section{Comportamento da tensão de cisalhamento do CAP 50/70 puro e modificado CM1}

A Figura 3 mostra o comportamento da tensão de cisalhamento com a taxa de cisalhamento do CAP 50/70 puro e modificado CM1, nas temperaturas de $60^{\circ} \mathrm{C}$ e de $170^{\circ} \mathrm{C}$. É observado nesta figura mudança de comportamento de fluido Newtoniano do CAP 50/70 para fluido nãoNewtoniano com a adição do modificador na temperatura de usinagem e compactação $170^{\circ} \mathrm{C}$, devido à mudança de tendência de a curva passar pela origem, concordando com dados da literatura para ligantes modificados [22]. É observado ainda que nesta temperatura de usinagem e compactação o ligante modificado apresenta comportamento de fluido do tipo plástico ideal. Entretanto, na temperatura de $60^{\circ} \mathrm{C}$ observa-se que ambos, o CAP 50/70 e o modificado CM1, apresentam comportamento de fluido não-Newtoniano.

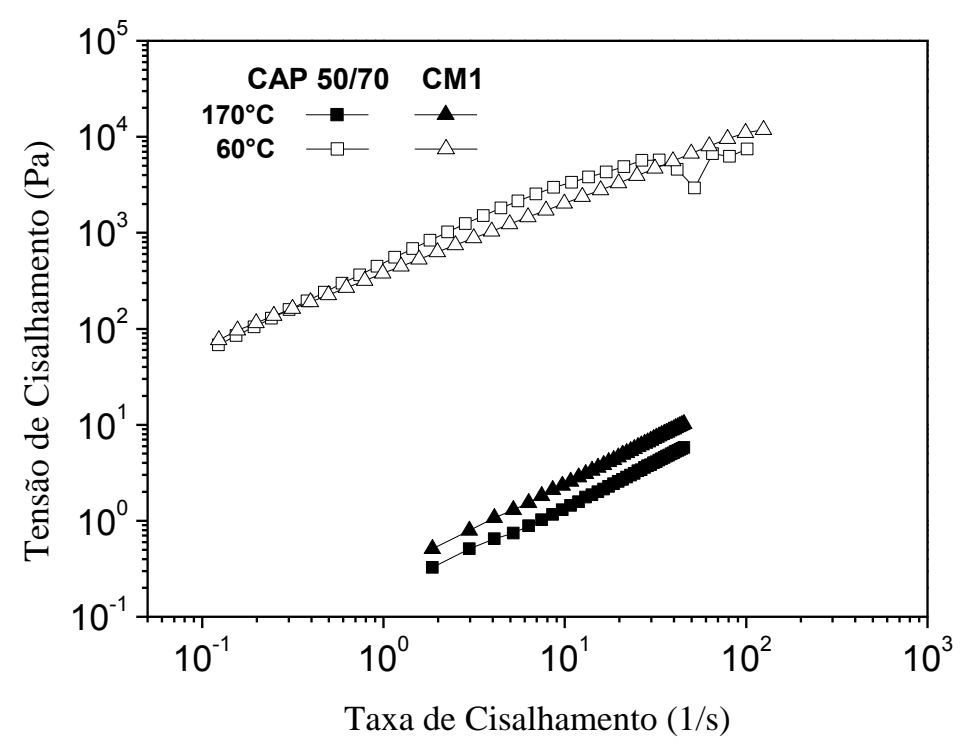

Figura 3 - Comportamento da tensão de cisalhamento com a taxa de cisalhamento de amostra de CAP 50/70 e de CMI, nas temperaturas de $60^{\circ} \mathrm{C}$ e $170^{\circ} \mathrm{C}$. 
A Figura 4 mostra o comportamento da viscosidade aparente com a taxa de cisalhamento do CAP 50/70 puro e modificado CM1, nas temperaturas de $60^{\circ} \mathrm{C}$ e de $170^{\circ} \mathrm{C}$. Observa-se que o comportamento da viscosidade corrobora os resultados obtidos dos dados da curva de tensão com a taxa de cisalhamento. Verifica-se a formação de um platô Newtoniano para o CAP 50/70 puro e modificado $\mathrm{CM} 1$ na temperatura de $170^{\circ} \mathrm{C}$. Para a temperatura de $60^{\circ} \mathrm{C}$ verificou-se formação de platô Newtoniano na amostra de CAP 50/70 em baixas taxa de cisalhamento, em contrapartida, para a amostra de modificado CM1, o mesmo apresenta comportamento de fluido pseudoplástico, corroborado pela literatura [7].

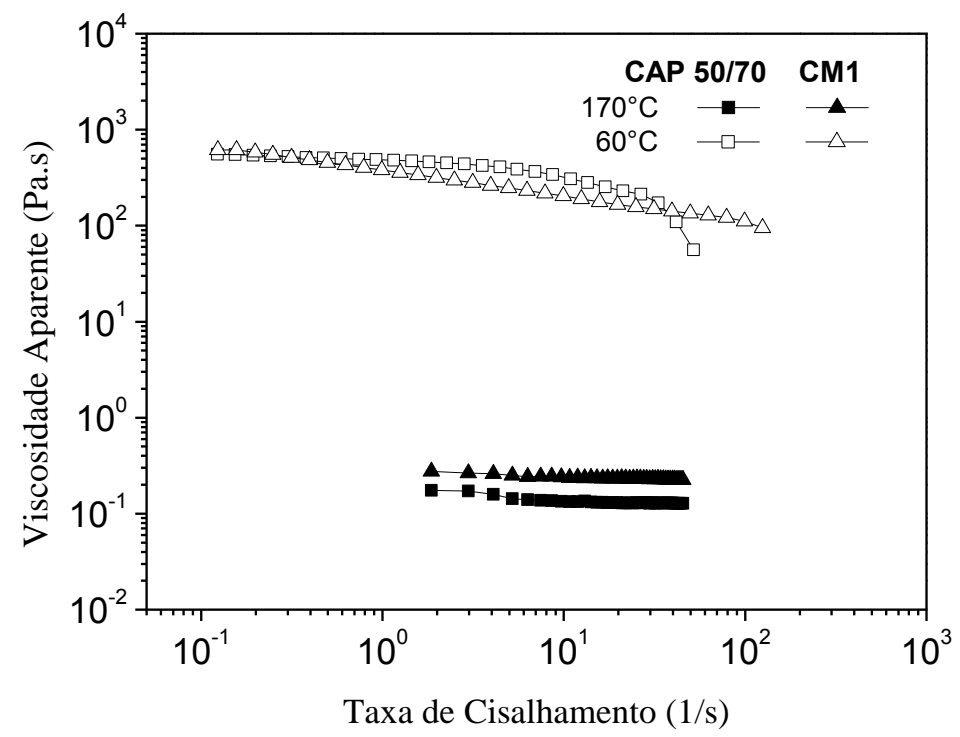

Figura 4 - Comportamento da viscosidade aparente com a taxa de cisalhamento de amostra de CAP $50 / 70$ e de $\mathrm{CM1}$, nas temperaturas de $60^{\circ} \mathrm{C}$ e $170^{\circ} \mathrm{C}$.

\section{Comportamento do módulo G' 'e G' do CAP 50/70 puro e modificado}

A Figura 5 mostra o comportamento do módulo elástico $G^{\prime}(\omega)$ e do módulo viscoso G' $(\omega)$ em função da frequência do CAP 50/70 puro e modificado CM1, na temperatura de $60^{\circ} \mathrm{C}$. Observa-se que a adição dos modificadores ao CAP 50/70 aumentou os módulos elástico e viscoso das misturas, contudo, com uma maior aproximação entre eles, o que significa melhor resistência à deformação permanente em toda faixa de frequência em estudo e que não há variação significativa com a variação da concentração do aditivo modificador do ligante.

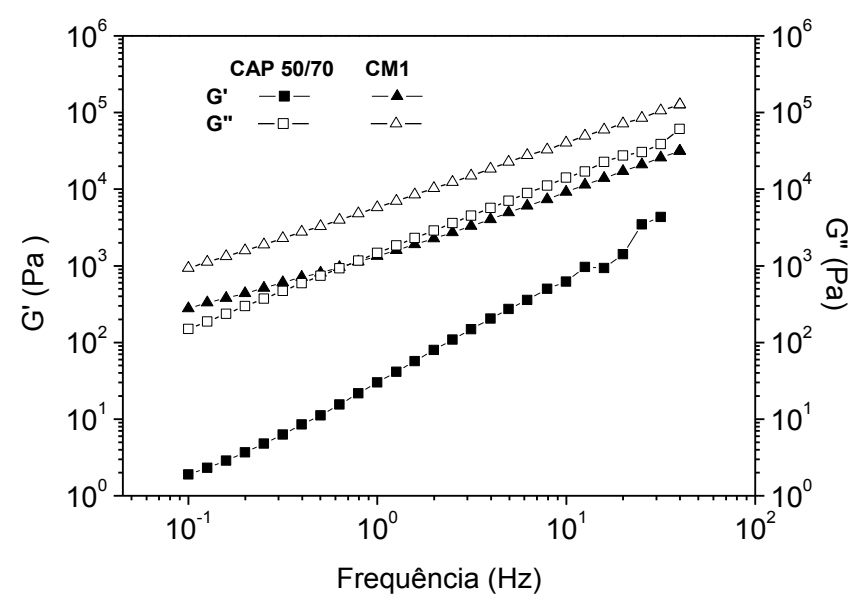


Figura 5 - Comportamento dos módulos elástico $G^{\prime}(\omega)$ e viscoso $G$ '” $(\omega)$ em função da frequência na temperatura de $60^{\circ} \mathrm{C}$ do CAP 50/70 e do CAP modificado CM1

\section{Avaliação da estabilidade de estocagem do CAP 50/70 modificado (CM1)}

A Figura 6 mostra comparativamente o comportamento da viscosidade aparente com a taxa de cisalhamento, na temperatura de $60^{\circ} \mathrm{C}$, do CAP 50/70 puro e modificado $\mathrm{CM} 1$ de amostras retidas do fundo e do topo do artefato de armazenamento, após resfriamento do ensaio de estocagem. Observa-se que a amostra de topo apresentou comportamento semelhante ao do CAP 50/70, isto é, de fluido newtoniano, enquanto que a amostra do fundo apresentou comportamento de fluido não newtoniano. A razão para tal comportamento pode estar no fato do agente modificante ser borra oleosa de petróleo advinda de tratamento primário óleo e nela existir material silicoso de densidade maior que a do ligante em estudo [12, 13].

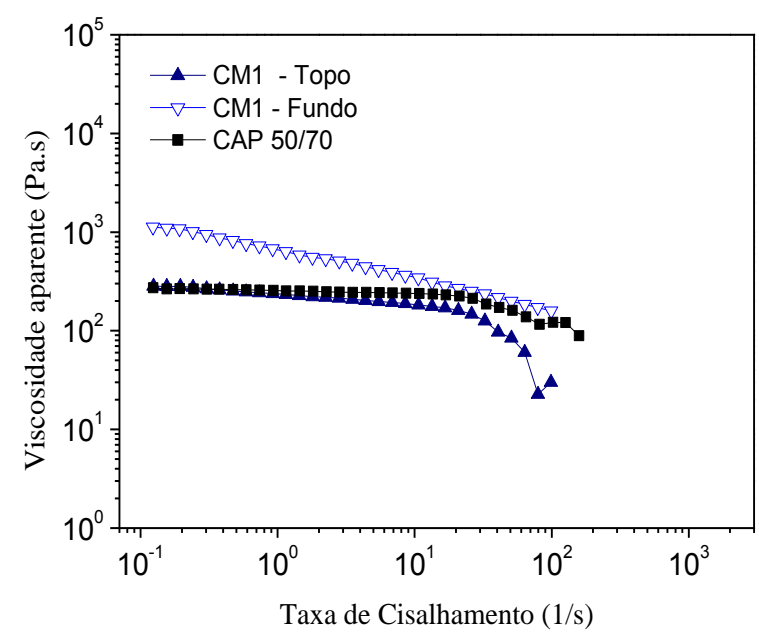

Figura 6-Comportamento da viscosidade aparente com a taxa de cisalhamento de amostra de CAP 50/70 e de CM1, na temperatura de $60^{\circ} \mathrm{C}$.

\section{CONCLUSÃO}

O comportamento térmico obtido por DSC da amostra de CAP 50/70 modificado mostrou que fase cristalina foi introduzida ao referido ligante modificado, e que a sua presença pode estar relacionada à presença de fenômeno de quimissorção entre o CAP 50/70 e componentes cristalizáveis presentes na borra oleosa. $\mathrm{E}$, os resultados das análises reológicas, na temperatura e $60^{\circ} \mathrm{C}$, em regime oscilatório e cisalhante, respectivamente, mostraram que a adição do modificante M1 aumentou os módulos de armazenamento ( $\left.G^{\prime}\right)$ e de viscosidade (G'), com maior aproximação entre eles, em toda faixa de frequência estudada, enquanto que o comportamento da viscosidade das amostras após 48 horas de estocagem revelou a existência de separação de fase, visto que a viscosidade da amostra do topo se equivale à amostra do CAP 50/70 puro, enquanto a amostra do fundo apresentou maior viscosidade, característica da borra oleosa de petróleo em estudo, advinda de tratamento primário de petróleo e nela existir frações de hidrocarboneto de alto peso molecular e material silicoso. Os resultados obtidos mostram que o referido ligante modificado atende às especificações brasileiras, no que se refere à temperatura de usinagem e compactação de misturas do tipo CAUQ, empregadas em revestimentos de pavimento rodoviário. Contudo, há separação de fase no ligante modificado na concentração dos modificadores estudados, tornando necessários estudos complementares sobre estabilidade de estocagem, pois torna necessária a adição de agentes de compatibilização para que o CAP 50/70 modificado com blenda de borra oleosa de petróleo advinda de tratamento primário de petróleo possa ser usado em mistura para revestimento de pavimento rodoviário. 


\section{AGRADECIMENTOS}

Os autores agradecem à EMURB - Aracaju/SE e a PETROBRAS/UO - SEAL pelas amostras de CAP 50/70 e borra oleosa, respectivamente, e a Coordenação de Aperfeiçoamento de Pessoal de Nível Superior (CAPES) pela bolsa recebida durante a realização do Curso de Mestrado em Engenharia Química no PEQ/UFS.

\section{REFERÊNCIAS BIBLIOGRÁFICAS}

1. Peiliang Cong, Weihua Luo, Peijun Xu, Hua Zhao. Investigation on recycling of SBS modified asphalt binders containing fresh asphalt and rejuvenating agents. Construction and Building Materials. 2015 Aug.; 91:225-231, doi:10.1016/j.conbuildmat.2015.05.041.

2. Yang Kang, Mingyu Song, Liang Pu, Tingfu Liu. Rheological behaviors of epoxy asphalt binder in comparison of base asphalt binder and SBS modified asphalt binder. Construction and Building Materials. 2015 Feb.; 76:343-350, doi:10.1016/j.conbuildmat.2014.12.020.

3. Jianming Wei, Zhangyong Liu, Yuzhen Zhang. Rheological properties of amorphous poly alpha olefin (APAO) modified asphalt binders. Construction and Building Materials. 2013 Nov.; 48:533-539, doi:10.1016/j.conbuildmat.2013.07.087.

4. Xiangyong Li, Chunfa Ouyang, Ye Yuan, Qun Gao, Kangsheng Zheng, Jun Yan. Evaluation of ethylene-acrylic acid copolymer (EAA)-modified asphalt: Fundamental investigations on mechanical and rheological properties. Construction and Building Materials. 2015 Aug.; 90:44-52, doi:10.1016/j.conbuildmat.2015.04.049.

5. Behnam Golestani, Boo H. Nam, Fereidoon M. Nejad, Shahab Fallah. Nanoclay application to asphalt concrete: Characterization of polymerand linear nanocomposite-modified asphalt binder and mixture Construction and Building Materials. 2015 Aug; 91:32-38, doi:10.1016/j.conbuildmat.2015.05.019.

6. RuienYu, Changqing Fang, Pei Liu, Xiaolong Liu, Yan Li. Storage stability and rheological properties of asphalt modified with waste packaging polyethylene and organic montmorillonite. Applied Clay Science. 2015 Feb.; 104: 1-7, doi:10.1016/j.clay.2014.11.033.

7. Feng Zhang, Jianying Yu, Shaopeng Wu. Effect of ageing on rheological properties of storage-stable SBS/sulfur-modified asphalts. Journal of Hazardous Materials. 2010 Oct.; 182 (1-3):507-517, doi:10.1016/j.jhazmat.2010.06.061.

8. Zahid Hossain, Biswajit Bairgi, Mark Belshe. Investigation of moisture damage resistance of GTRmodified asphalt binder by static contact angle measurements. Construction and Building Materials. 2015 Oct.; 95:45-53, doi:10.1016/j.conbuildmat.2015.07.032.

9. Davide Lo Presti. Recycled Tyre Rubber Modified Bitumens for road asphalt mixtures: A literature review. Construction and Building Materials. 2013 Dec.; 49:863-881, doi:10.1016/j.conbuildmat.2013.09.007.

10. Xiang Shu, Baoshan Huang. Recycling of waste tire rubber in asphalt and portland cement concrete: An overview. Construction and Building Materials. 2014 Sept.; 67:217-224, doi:10.1016/j.conbuildmat.2013.11.027.

11. Peiliang Cong, Xiao Wang, Peijun Xu, Jianfei Liu, Rui He, Shuanfa Chen. Investigation on properties of polymer modified asphalt containing various antiaging agents Polymer Degradation and Stability. 2013 Dec.; 98:262 - 263, doi:10.1016/j.polymdegradstab.2013.09.024.

12. Guangji Hua, Jianbing Li, Guangming Zeng. Recent development in the treatment of oily sludge from petroleum industry: A review. Journal of Hazardous Materials. 2013 Oct.; 261:470-490, doi:10.1016/j.jhazmat.2013.07.069.

13. Sanaa Jamaly, Adewale Giwa, Shadi Wajih Hasan. Recent improvements in oily wastewater treatment: Progress, challenges, and future opportunities. Journal of Environmental Sciences. 2015 Nov.; 37(1):15-30, doi:10.1016/j.jes.2015.04.011.

14. Ming Liang, Xue Xin, Weiyu Fan, Huadong Sun, Yan Yao, Baodong Xing. Viscous properties, storage stability and their relationships with microstructure of tire scrap rubber modified asphalt. Construction and Building Materials. 2015 Jan.; 74:124-131, doi:10.1016/j.conbuildmat.2014.10.015.

15. Ming Liang, Xue Xin, Weiyu Fan, Hui Luo, Xiaobo Wang, Baodong Xing. Investigation of the rheological properties and storage stability of CR/SBS modified asphalt. Construction and Building Materials. 2015 Jan.; 74:235-240, doi:10.1016/j.conbuildmat.2014.10.022.

16. Jublee Jasmine, Suparna Mukherji. Characterization of oily sludge from a refinery and biodegradability assessment using various hydrocarbon degrading strains and reconstituted consortia. Journal of Environmental Management. 2015 Feb.; 149:118-125, doi:10.1016/j.jenvman.2014.10.007. 
17. Ju Zhang, Jianbing Li, Ronald Thring, Lei Liu. Application of ultrasound and Fenton's reaction process for the treatment of oily sludge. Procedia Environmental Sciences. 2013; 18: 686-693, doi:10.1016/j.proenv.2013.04.093.

18. B.C.A. Pinheiro, J. N. F. Holanda. Obtainment of porcelain floor tiles added with petroleum oily sludge. Ceramics International. 2013 Jan.; 39(1): 57-63, doi:10.1016/j.proenv.2013.04.093.

19. B. Barra, L. Momma, Y. Guerrero, L. Bernucci. Fatigue behavior of dense asphalt mixes in dry and environmental-conditioning states. Construction and Building. 2012 Apr; 29:128-134, doi:10.1016/j.conbuildmat.2011.10.003.

20. Gabrielle Fritschy, Eugène Papirer. Interactions between a bitumen, its components and model fillers. Fuel, 1978 Nov.; 57(11): 701-704, doi:10.1016/0016-2361(78)90025-X.

21. Antonio Greco, Alfonso Maffezzoli. Polymer Testing. Correction of melting peaks of different PE grades accounting for heat transfer in DSC samples. 2008 Feb.; 27:61-74, doi:10.1016/j.polymertesting.2007.09.001.

22.F. Cardone, G. Ferrotti, F. Frigio, F. Canestrari. Influence of polymer modification on asphalt binder dynamic and steady flow viscosities. Construction and Building Materials. 2014 Nov.; $71: 435$ - 443, doi:10.1016/j.conbuildmat.2014.08.043. 